\title{
Design and Implementation of Mobile Intelligent Terminal Network Communication in Smart Home
}

\author{
Shulin Yang ${ }^{1}$, Nan Yang ${ }^{2}$, Jieping $\mathrm{Hu}^{3}$ and Guyang Wang \\ ${ }^{1}$ College of Information Engineering, Beijing Institute of Graphics Communication, Beijing, China \\ ${ }^{2}$ General Education College, Beijing International Studies University \\ ${ }^{3}$ Department of Basic, Beijing Institute of Graphics Communication, Beijing, China
}

\begin{abstract}
Aiming at the demand of smart home application, the smart home control system is analyzed and researched. We proposed a low cost and expandable home control system as well as the network communication solution. Based on the Android mobile phone platform or tablet computer, a set of control system is designed for smart home. The various resources provided by Android is used to establish the smart home control terminal software, and the microcontroller is used to develop a smart home control gateway. Through the effective cooperation of the mobile smart terminals, servers, home gateway and front-end controller, the remote control and local control of household appliances are realized. The proposed system can be used for ordinary residential home users and can also be used for hotel subsystems. The results show that the hardware of the system is simple, low-cost, reliable and easy to expand.
\end{abstract}

Keywords-smart home; android; home gateway; network contro I mode; communication protocol

\section{INTRODUCTION}

The birth of smart home makes people's life has improved comfort, safety and security has been greatly improved the quality of life [1-2]. The combination and development of computer technology, network technology and mobile communication technology provides the premise and condition for the rapid development of smart home[3]. With the popularity of intelligent terminal, smart mobile devices as a smart home control terminal has gradually become the development direction of smart home system, smart mobile devices in addition to can bring convenience to the user and the good human-machine interface will be real smart home into a "people-oriented" modern household environment.

In this paper, considering the breadth of Android system applications and development trend of smart home to App runs on Android intelligent mobile devices to control the terminal, in order to make the STM32-based home gateway controller and distribution of the front control of each room in the home Sites control home appliances, and use the Internet to connect to the server for remote control, designed a low-cost, reliable and practical, user-friendly smart home system.

\section{SyStem StRUCTURE AND NeTWORK CONTROL MODE}

\section{A. System Structure}

The smart home system mainly consists of mobile intelligent terminals, server, home gateway, and front controllers located in each room. This system structure is shown in Figure 1. Intelligent terminal operated by the user, is responsible for sending commands; Server as the mobile control of the bridge between the intelligent terminal and family gateway, is responsible for communication between them; Home gateway after receive commands from the server or intelligent terminal, forwarded to corresponding front controller; The front controller, as the ultimate executor of the command, controls the operation of device.

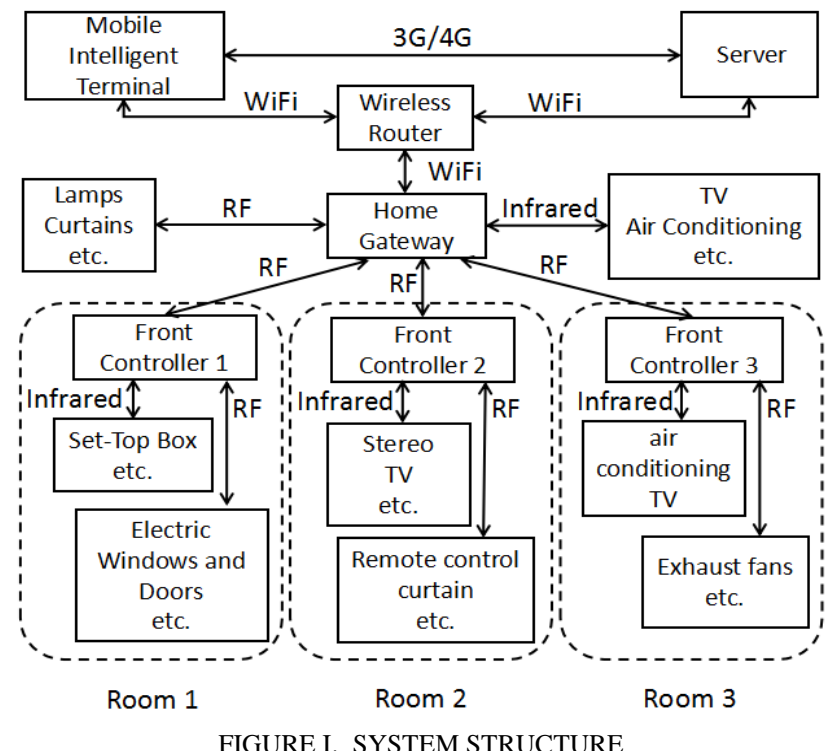

\section{B. Network Control Mode}

Smart home system control method is divided into two kinds: One is the internal network control mode that user use home WiFi connect to home gateway, then control front device by the home gateway; Another is the external network control mode that user first by $3 g / 4 g$ network access server which find the corresponding family gateway, and then by the gateway control household appliances.

1) Internal network control mode: Mobile terminal send control instructions to the home gateway by WiFi , home gateway after receiving the control command, the control instruction is resolved, and the family gateway is determined according to the instruction that the home gateway is forwarded to the corresponding front end controller or directly controls the work of the corresponding household electric appliance. Front controller distributed in each room home is mainly responsible for receiving RF radio frequency control signal from the home 
gateway, and the signal into control instruction, is used to control the household appliances. Household appliances after receiving control instructions, perform related functions, and equipment status information back to control terminal, so as to realize the real time intelligent control of smart phone or iPad to household appliances[4]. The working process of the internal network control is shown in Figure 2.

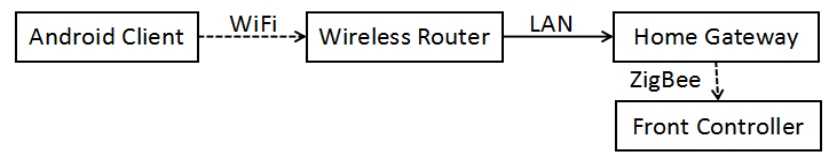

FIGURE II. FLOW CHART OF INTERNAL NETWORK CONTROL

2) External network control mode: The mobile intelligent terminal through $3 \mathrm{G}$ or $4 \mathrm{G}$ network to connect to the server, realize the remote communication with the server, the server is responsible for finding the home gateway corresponding to the client account, and communicate with the home gateway via Internet finally, to achieve control of household appliances through home gateway. External control is good as long as the user uses the client to access the Internet, you can to control household appliances anytime and anywhere, no longer subject to the influence of the geographical position. The working process of the external network control is shown in Figure 3.

\begin{tabular}{|c|c|c|c|c|}
\hline Android Client & $3 G / 4 G$ & Server & Internet & Wireless Router \\
\hline & & & & LAN \\
\hline & & Front Controller & & Home Gateway \\
\hline
\end{tabular}

FIGURE III. FLOW CHART OF EXTERNAL NETWORK CONTROL

\section{HOME GATEWAY AND FRONT CONTROLLER}

The difference between home gateway and front controller is that home gateway has a WiFi modules, and front control has no. The home gateway is composed of STM32F101C8 main controller, infrared remote control transmitting and receiving module, $315 \mathrm{MHz}$ and $433 \mathrm{MHz}$ radio frequency transmitting and receiving module, WiFi module, temperature and humidity sensor, smoke sensor and other components:

1) WiFi transceiver circuit module: The module is mainly responsible for data interaction with intelligent terminal App or server. WiFi module successfully connected to the home router, you can communicate with the terminal App or server through LAN.

2) $R f$ signal transceiver module: The module mainly realizes the data exchange between home gateway and front controller, the communication network of household and Learning function of the home gateway to RF remote control signal. The RF remote control signal of Household appliances is mainly the radio frequency signal of $433 \mathrm{MHz}$ and $315 \mathrm{MHz}$ frequency band.

3) Infrared signal receiving and sending module: The module mainly realize the infrared home appliances control and learning function of infrared remote control signal and other functions.
4) Sensor module: Smoke sensor used to collect room smoke information to determine whether there is a fire; Temperature and humidity sensors to collect the information such as temperature, humidity room.

\section{THE DESIGN OF THE INTELLIGENT TERMINAL NETWORK COMMUNICATION MODULE}

The network communication module of the intelligent terminal is responsible for the data exchange between the intelligent terminal and the home gateway or the home server, and is the basis of all the function modules. Internal network control adopts TCP protocol, external network control adopts HTTPS or WebService protocol. In order to adapt to different family scene, facilitate communication between mobile intelligent terminal with gateway, use the UDP protocol to obtain first gateway IP and port, namely mobile intelligent terminal sends a request to the home gateway by UDP datagram, to obtain the IP address of the home gateway and listening port number. IP multicast technology is used in the concrete realization[5].

The mobile intelligent terminal uses the IP address and the monitoring port to construct the Socket and establish the TCP connection width the home gateway module . For communication security, use SSLSocket. After the connection is successful, the mobile intelligent terminal and the gateway service module can use the TCP connection to transfer the data to each other. The network control use HTTPS or WebService protocol to communicate ommunicate with the home server via Internet, And then server communicate with the home gateway server, in order to achieve the remote control. The hierarchical structure of class is shown in Figure4.

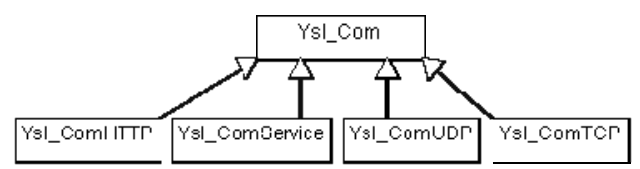

FIGURE IV. CLASS HIERARCHY

Ysl_Com class encapsulates the context and handler, some constants, and method to operate sessionId and factory method to create secure socket . Ysl_ComUDP, Ysl_ComTCP, Ysl_ComHTTP, Ysl_ComService classes are inherited Ysl_Com, respectively for the UDP protocol communication, TCP communications, HTTP protocol communication and WebService communication, adopt the singleton pattern. Among them, HTTP communication using HttpClient Apache to achieve [6], and its expansion, in order to achieve the TSL protocol communication [7]; WebService communication using Ksoap2 to achieve [8].

\section{DESIGN OF COMMUnicAtion Protocol}

Upload data using an array of bytes, the returned data using JSON format. Upload the data, a total of 60 bytes As shown in Table I.

TABLE I. COMMUNICATION PROTOCOL FORMAT OF SYSTEM

\begin{tabular}{|l|l|l|l|}
\hline serialNo & deviceId & instructionName & instructionValue \\
\hline
\end{tabular}


1) Serialno: intelligent terminal serial number, 20 bytes.

2) Deviceid: household appliance ID, 5 bytes

3) Instructionname: instruction, 20 bytes.

4) Instructionvalue: instruction value, 15 bytes.

Receive data using JSON format, for example: $\{"$ instructionName ":" run_state ", "instructionValue" : "on"\}. When parsing the JSON data, mainly use JSONObject or JSONArray.

\section{CONCLUSION}

This paper designed a set of intelligent household system $b$ ased on Android, Android smart phone or iPAD as control ter minal obvious advantages; Design and development of applica tion software can be installed and used on Android operating s ystem platform, easy to promote; Rf wireless communication $t$ echnology as a means of home network communication syste $\mathrm{m}$ cost is reduced. But not commonly used for smart appliance $\mathrm{s}$ and socket etc., can be defined through intelligent control pa nel and special Settings. Proven by actual test, the system can be implemented using the user's smart phone or iPAD instead of all household appliances remote control, real-time monitori ng and control the function of the household electrical applian ces, has brought great convenience to People's Daily life.

\section{ACKNOWLEDGMENT}

This paper was supported by National Natural Science Fou ndation "Analysis and Solution of Nonlinear Dynamic Process in High Speed Printing Equipment”(No.61272030) and “online ar Dynamics model and Analysis of Gear Systems for Offset Pr ess” (No. 61472461).

\section{REFERENCES}

[1] Xiang Jun, Xie Zhanfu, "The smart home system based on embedded Int ernet/Intranet model and implementation”, Computer engineering and de sign, 2005, Vol.26, 9, pp.167-169.

[2] Shao Pengfei, Wang Ji, Zhang Baoru, "Smart home system in mobile Int ernet research”, Computer measurement and control, 2012, Vol.20, 2, pp. 474-476, 479.

[3] S Kumar, "Ubiquitous smart home system using Android application”,In ternational Journal of Computer Networks \& Communications, 2014, Vo 1.6, 1, pp.33-43.

[4] Reto Meier, Professional Android 2 Application Development, tsinghua university press, 2010.

[5] Android development: Multicast (multicast) and broadcast. http://gqdy36 5.iteye.com/blog/1992584.

[6] Apache HttpClient. http:/hc.apache.org/httpclient-3.x/.

[7] Yang Shulin,Hu Jieping. On Double-sided Secure CommunicationTechn ology in Android Platform. Journal of Beijing Institute of Graphic Com mun ication, 2013, Vol.21, 4, pp.8-10.

[8] Java Web Services: Metro Introduction. http://www. ibm. com/developer works/cn /java/j-jws9. htm. 